\title{
SOFTWARE-DEFINED MICROSTRIP ANTENNAS ENABLED THROUGH LARGE VERTICAL DISPLACEMENT ZIPPER MICROACTUATORS
}

\author{
Jason Felder ${ }^{1,2}$, Eugene Lee ${ }^{2}$, Eric Walton ${ }^{3}$, Don L. DeVoe ${ }^{l}$ \\ ${ }^{1}$ Dept. of Mechanical Engineering, University of Maryland, College Park, MD, USA \\ ${ }^{2}$ Syntonics LLC, Columbia, MD, USA \\ ${ }^{3}$ Dept. of Electrical and Computer Engineering, The Ohio State University, Columbus, OH, USA
}

\begin{abstract}
This paper describes a novel large-displacement electrostatic "zipper" microactuator capable of achieving hundreds of microns of out-of-plane deflection, and application of fabricated microactuators to the control of millimeter-scale microfabricated capacitive pixels enabling "software-defined" microstrip antennas. The software-defined microstrip patch antenna concept relies on a reconfigurable array of individually addressed pixels. Current antenna designs demand pixel footprints below $1 \mathrm{~mm}^{2}$, with 300 $\mu \mathrm{m}$ vertical deflections, $1 \mathrm{~ms}$ response times, and low power consumption. To address this challenge we have developed a new technology based on electrostatic zipper actuation, in which a curved beam is switched between bistable states by application of a voltage across a dielectric film separating the beam from a flat electrode surface. This technology is novel in its use of $\mathrm{SiO}_{2}$ as both a high quality dielectric and the stressed layer of the bimorph providing curved actuation, enabling large out-of-plane deflection and large forces.
\end{abstract}

\section{INTRODUCTION}

Despite decades of research on large-displacement out-ofplane microactuation, including electrothermal [1], thin film metallic glass [2], and asymmetric comb drive actuators [3], established technologies are inadequate to meet the required demands of a microstrip patch antenna: footprints smaller than $1 \mathrm{~mm}^{2}, 1 \mathrm{~ms}$ response time, and a tip deflection of at least $300 \mu \mathrm{m}$.

Bistable electrostatic actuators, termed "Zipper" actuators, have been fabricated that are capable of meeting the design requirements [4-6]. The theory and mathematics behind electrostatic zipper actuators has been well documented and can be found in numerous publications such as [7] and [8]. All, however, are metallic bimorph structures, which can become expensive with gold, and which require unnecessarily complex fabrication processes. [5] report a zipper-style bimorph actuator made of 2.3 $\mu \mathrm{m}$ of soft gold and $0.2 \mu \mathrm{m}$ of hard gold, requiring an eleven step fabrication process (photolithography steps were not counted), the final step of which is a release etch of a sacrificial layer, leading to issues with stiction. Dhaubanjar et al. report a poly-Si and gold bimorph beam fabricated using the MUMPS process, and ending in an HF release etch [4]. Chang et al. demonstrate a chromium and aluminum bimorph that requires four materials, five photolithography masks, and nine steps to fabricate, one of which is a timed RIE etch, which can ruin the device if prolonged [7]. Others have shown similar metallic bimorph actuators with comparably complex fabrication processes [6].

We propose a simple new technology based on electrostatic zipper actuation [9], in which a curved beam is switched between bistable states by application of a voltage across a dielectric film separating the beam from a flat electrode surface. Unlike existing thin film zipper microactuators, the present devices comprise bimorph beams fabricated from an SOI wafer with a $10 \mu \mathrm{m}$ active layer $(\mathrm{Si})$ and $2 \mu \mathrm{m}$ thick thermal oxide $\left(\mathrm{SiO}_{2}\right)$. Compressive stress in the $\mathrm{SiO}_{2}$ results in well-defined initial beam curvature, while also serving as a high quality and relatively high-k dielectric for electrostatic actuation. Similarly, the thickness of the beam, relative to most other zipper actuators, provides for high forces at the tip. The novel use of $\mathrm{SiO}_{2}$ for both curvature control and dielectric isolation provides significantly larger deflections than traditional zipper actuators while reducing pull-in voltages. Additionally, since the dielectric layer is part of the actuated beam any flat conductive surface (such as a doped $\mathrm{Si}$ wafer) can be used as the underlying electrode without any fabrication. Lastly, using only two common, and well-characterized materials results in a simple, well-defined, four-step fabrication process, that is achieved using only two masks, and could, theoretically be achieved using only one.

\section{DEVICE FABRICATION}

The fabrication of the microactuators is a four step process, illustrated in Fig. 1. A p-type (100) SOI, with a $10 \mu \mathrm{m}$ thick active Si layer, a $1 \mu \mathrm{m}$ thick buried oxide (BOX) layer, and a $500 \mu \mathrm{m}$ thick handle $\mathrm{Si}$ layer, serves as the base for the actuator fabrication, as shown in Fig. 1( $a$ ). To start, $2 \mu \mathrm{m}$ of thermal silicon dioxide is grown to serve as the dielectric and the stressor to induce beam bending (Fig. 1(b)). Next, the frontside $\mathrm{SiO}_{2}$ and $\mathrm{Si}$ are patterned using DRIE to define the actuator beams, as shown in Fig. 1(c). In this 5 minute etch process, the BOX layer serves as an etch stop for the frontside DRIE. Since the etch rate ratio of Si: $\mathrm{SiO}_{2}$ for this particular process was characterized as 50:1 (2.5 $\mu \mathrm{m} / \mathrm{min}$ and $0.05 \mu \mathrm{m} / \mathrm{min}$ ), and the active $\mathrm{Si}$ is only $10 \mu \mathrm{m}$ thick, the Si can be safely etched, with a $25 \%$ over etch without worry of damaging the BOX. The backside $\mathrm{SiO}_{2}$ is removed using RIE and the backside $\mathrm{Si}$ is then etched using the same DRIE process to open backside access down to the BOX, as shown in Fig. 1(d). In

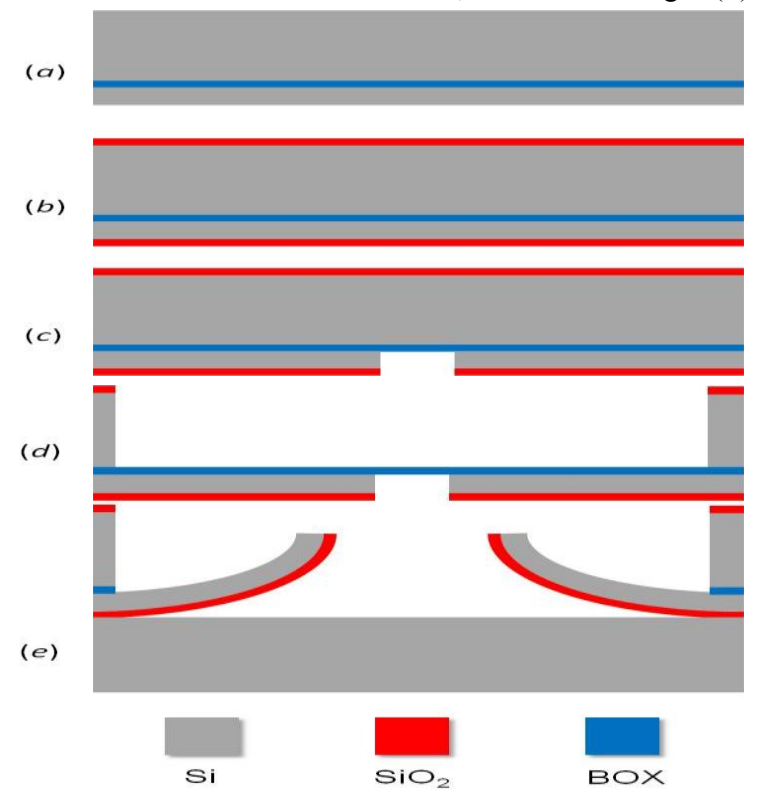

Figure 1: Fabrication process for zipper actuators using one mask and four process steps. 
the fourth and final step, the beams are released in Fig. 1(e) using RIE to remove the BOX and affixed to an underlying doped $\mathrm{Si}$ electrode. The stress induced by the $\mathrm{SiO}_{2}$ causes the beam to curve upon release. In the final iteration of the actuator fabrication, the entire backside will be removed using a buffered oxide etch (BOE) of the BOX layer, and the beams will be thermocompression bonded to the underlying electrode. However, for the purpose of this study, the actuators were mechanically clamped to the electrode.

\section{CHARACTERIZATION OF ZIPPER ACTUATORS}

Both the beam tip deflection, and the voltage necessary to "pull-in" the beam, $\mathrm{V}_{\mathrm{PI}}$, were characterized in this study. It is important to note the positive correlation between the two parameters. Beam deflection is defined by the radius of curvature of the beam. That is, as the radius of curvature becomes small, the beam gains deflection more quickly, achieving a greater tip deflection over a set beam length. $\mathrm{V}_{\mathrm{PI}}$ behaves similarly, requiring a greater voltage bias between the beam and underlying electrode to "pull-in" a beam with a smaller radius of curvature [9].

\section{Deflection Characterization}

The first set of cantilevers was fabricated with an $\mathrm{SiO}_{2}$ thickness of $1.7 \mu \mathrm{m}$. Fig. 2(a) shows a set of cantilevers (upside down) used for characterization and Fig. 2(b) shows a $1750 \mu \mathrm{m}$ fully released double cantilever beam. Optical defocusing, shown in the inset in Fig. 2(b), was used to characterize beam tip deflection. Deflection measurements taken for beams measuring $500,1000,1500$, and $2000 \mu \mathrm{ms}$ correlate well with an analytic model (Fig. 3) originally used to predict beam performance, and a COMSOL model used to corroborate results (Fig. 4). The measured deflections validate both the analytic and COMSOL models for use in future beam designs.

\section{Voltage Characterization}

A high voltage power source and a micro-stage with a digital microscope were used to apply and measure the required pull-in voltage, $\mathrm{V}_{\mathrm{PI}}$. Since the high voltage power source has a digital display accurate to $0.1 \mathrm{kV}$, a multimeter was used to obtain a more accurate voltage reading. The analytic model used above to predict beam deflection was used to predict $\mathrm{V}_{\mathrm{PI}}$ as well. Since $\mathrm{V}_{\mathrm{PI}}$ is a function of the beams radius of curvature, it is independent of the length and width of the beam. Hence, for a given bimorph beam with fixed $\mathrm{Si}$ and $\mathrm{SiO}_{2}$ thickness, $\mathrm{V}_{\mathrm{PI}}$ will be constant for any beam length. Fig. 5 shows measured $\mathrm{V}_{\mathrm{PI}}$ values for various beam lengths plotted alongside the constant $\mathrm{V}_{\mathrm{PI}}$ for three different values of the gap between the beam's base and the underlying electrode. As mentioned above, in this fabrication iteration, the fabricated actuators were mechanically clamped down to the underlying electrode. This can lead to variation in the gap height between the beam base and the electrode. Hence, Fig. 6 shows the analytic model $\mathrm{V}_{\mathrm{PI}}$ values for gap heights of 1,2 and $3 \mu \mathrm{m}$ 's, to demonstrate the validity of the measured $\mathrm{V}_{\mathrm{PI}}$ values. Although the measured values are relatively scattered, they all fall within reasonable values of $\mathrm{V}_{\mathrm{PI}}$ given the variability in gap height that can easily occur through mechanical clamping. In later iterations of actuator fabrication thermocompression bonding will be used to define a uniform gap height (ideally $2 \mu \mathrm{m}$ ) across all actuators. Nonetheless, the model and measured results illustrate respectable and acceptable "pull-in" voltages for beams of large deflections.
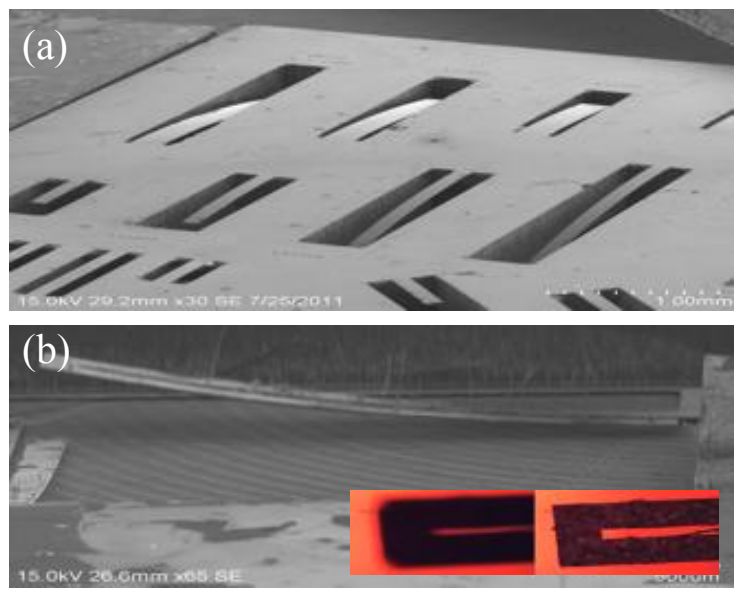

Figure 2: Single- and double-zipper cantilever actuators (a) before and (b) after bonding to the electrode layer. An optical defocusing measurement of beam deflection is shown inset.

Deflection at Tip of the Beam vs. Beam Length

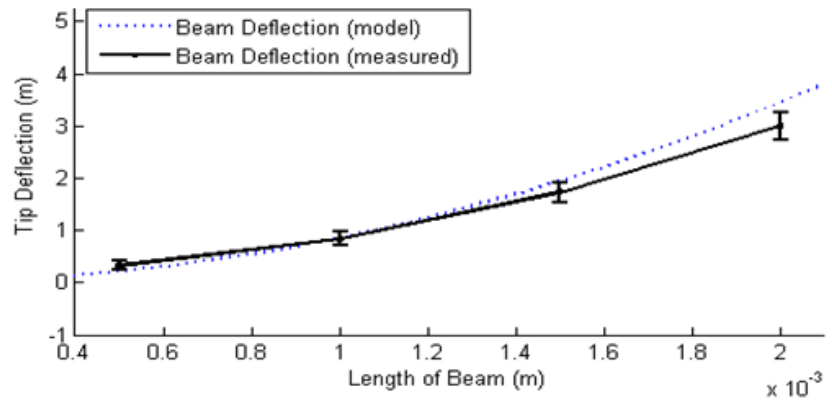

Figure 3: Agreement between analytic model for beam deflection, and actual beam deflection measured using optical defocusing.

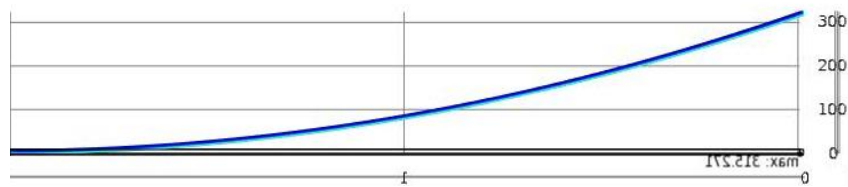

Figure 4: COMSOL model of $2000 \mu \mathrm{m}$ beam with total deflection of $315 \mu \mathrm{m}$, in correlation with analytic model, and measured deflection for 2000 um beam.

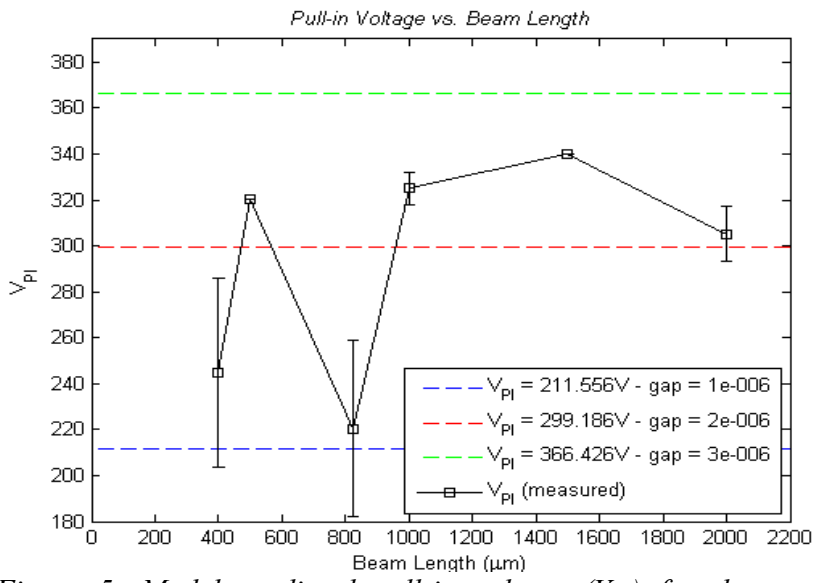

Figure 5: Model predicted pull-in voltage $\left(V_{P I}\right)$ for three gap values (dotted lines) plotted with measured values of $V_{P I}$ for a set of cantilever beam lengths. 


\section{APPLICATION IN MICROSTRIP PATCH ANTENNA}

The software-defined microstrip patch antenna concept relies on a reconfigurable array of individually addressed pixels (Fig. 6). Each pixel comprises a dielectric substrate sandwiched by conductive films, with the upper film serving as a waveguide and the lower film providing a ground plane. Pixels may be actively raised or lowered to define the desired microstrip circuit, enabling the formation of patch antennas that can scan in both frequency and angle by varying the size of the antennas or the feed network structure, or the formation of multiple antennas at different frequencies and polarizations. Current antenna designs demand pixel footprints below $1 \mathrm{~mm}^{2}$, with $300 \mu \mathrm{m}$ vertical deflections, $1 \mathrm{~ms}$ response times, and low power consumption.

Preliminary modeling determined that a linear beam, confined to the $1 \mathrm{~mm}^{2}$ footprint necessitated by the Microstrip Patch Antenna, would not be able to achieve the required $300 \mu \mathrm{m}$ deflection. A logical solution was to fabricate spiral actuators, thereby packing the greatest beam length into a $1 \mathrm{~mm}^{2}$ area. Spiral beams were simulated as linear beams, and the analytic model above was used to arrive at a beam length of at least $2000 \mu \mathrm{m}$ in order to reach $300 \mu \mathrm{m}$ tip deflection.

\section{Geometrical Challenge - Spiral Beams}

After fabrication of various spiral geometries, it became immediately clear that there was a fundamental issue with the

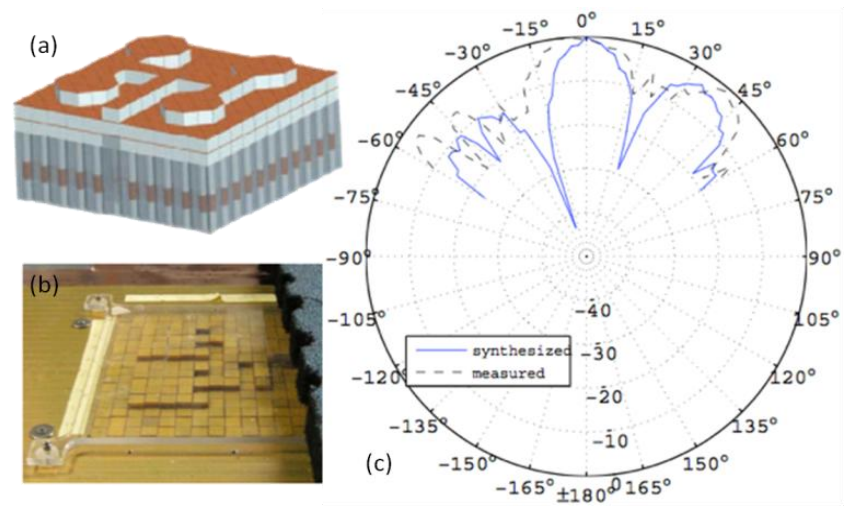

Figure 6: (a) Schematic and (b) photograph of a prototype software-defined antenna, and (c) radiation pattern at $8.3 \mathrm{GHz}$ measured using the prototype device.

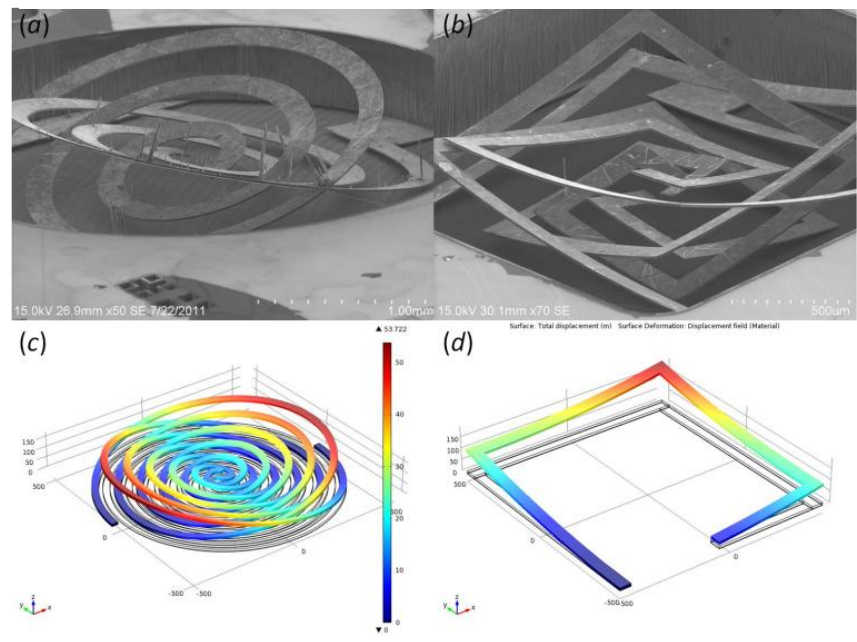

Figure 7: (a) Square spiral (b) round spiral and (c) COMSOL spirals, all exhibiting "bird-nest" phenomena. (d) COMSOL model illustrating the theory behind the bird-nest phenomenon. spiral concept. Unlike the spiral actuators in [2] where spiral actuators achieve a continuously rising slope, the actuators fabricated here achieved a maximum deflection exactly $180^{\circ}$ from the anchor point, no matter the shape (smooth spiral or square spiral) or configuration of the actuator beam. This "bird-nest" phenomenon is shown below in Fig. 7(a) and $(b)$, in SEM's of various spiral actuators, and validated by a COMSOL model of a similar spiral actuator in Fig. 7(c). The cause behind the "birdnest" is best illustrated by Fig. 7(d). Each successive "leg" of the continuous beam has initial conditions that match the tip conditions of the end of the leg to which it is attached. Hence, the third leg, positioned $180^{\circ}$ from the base of the continuous beam has an initial slope down towards the base that is the result of the initial conditions of the previous two legs. This idea can be applied to a spiral, which is essentially an infinite number of small "legs", and can be used to explain why the spiral's maximum deflection occurs $180^{\circ}$ from the base, no matter the configuration of the beams. In [2], the spiral actuators are given a deflection by pulling the spiral out of plane at high heat, a process that induces a natural torque in the spiral, so that as it rises continuously, its torque warps the beam, correcting for its natural downward slope that would occur after $180^{\circ}$. The fabrication of our actuators uses the natural stress of the $\mathrm{SiO}_{2}$ to provide beam deflection. Hence, no beam torque exists to counteract the natural downward slope as the spiral progresses.

\section{Solution to "Bird-Nest" Geometry Challenge}

In order to achieve $300 \mu \mathrm{m}$ deflections in a $1 \mathrm{~mm}^{2}$ footprint, serpentine actuators were fabricated in a modified process involving selective removal of oxide from portions of the beam. In this fabrication process, $2 \mu \mathrm{m}$ of $\mathrm{SiO}_{2}$ was grown atop the $10 \mu \mathrm{m}$ of Si. While a more complex fabrication process is required the resulting designs are capable of achieving exceptionally large deflections within a compact space. Moreover, the geometry lends itself to a symmetric design creating stability and culminating in a flat center area ideal for affixing an object, such as the pixel for the microstrip patch antenna.

A segment of the profile of the "Microstage" actuator can be seen in Fig. 8. The first leg consists of an actuated segment of length $L$ and a linear segment of length $1000-L$. The actuated segment is an $\mathrm{Si} / \mathrm{SiO}_{2}$ zipper actuator of the type presented above, followed by a linear segment of only Si whose slope and deflection amplification are defined by the slope at the end of the actuated segment. The second leg consists of an actuated segment of $2 L$ followed by a linear segment of $1000-2 L$.

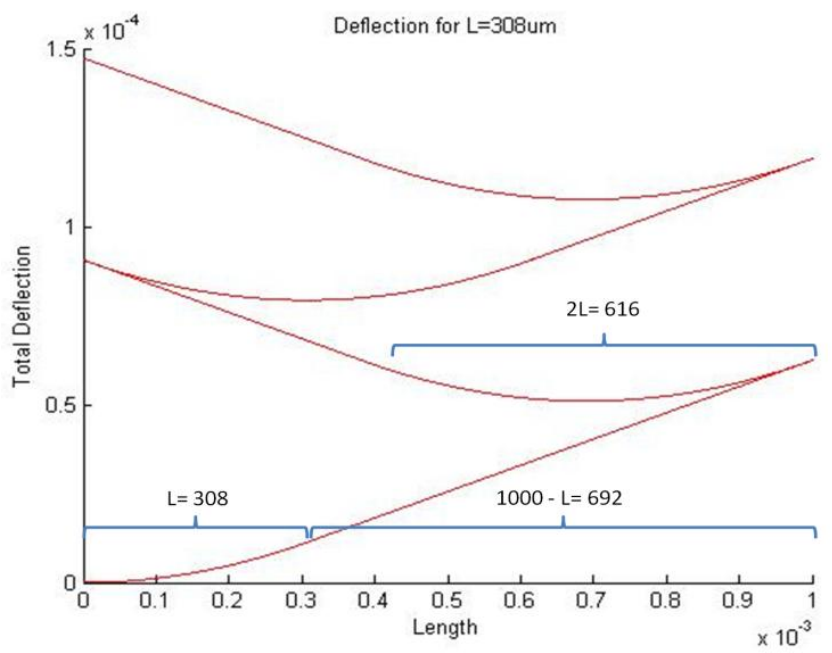

Figure 8: Analytic model of z-profile for a micro-stage actuator. 


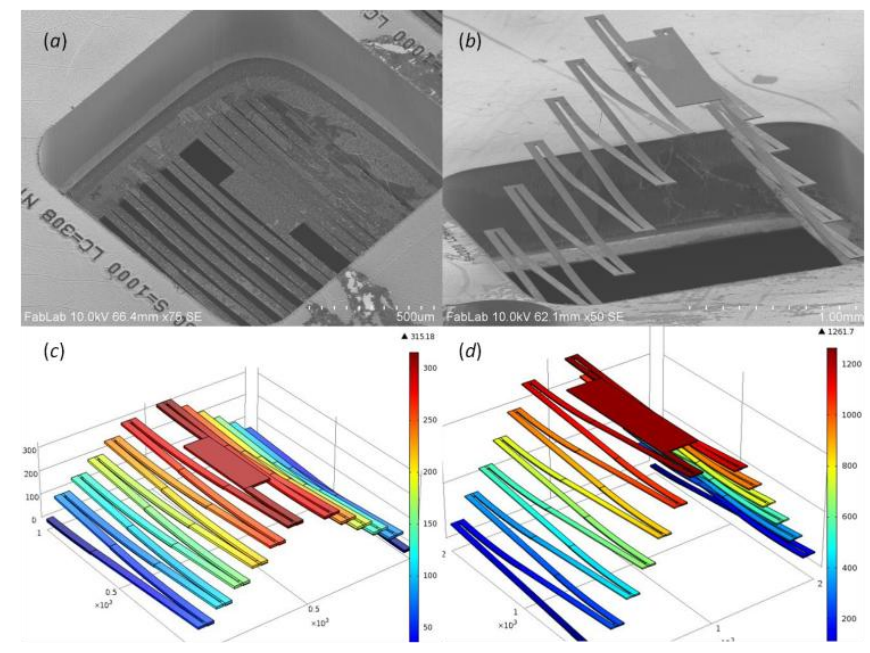

Figure 9: (a) Fully released $1 \mathrm{~mm}^{2}$ Microstage, with a stage deflection of $290 \mu \mathrm{m}$. (b) Fully released $2 \mathrm{~mm}$ Microstage, with a stage deflection of $1243 \mu \mathrm{m}$. (c) $\mathrm{A} \mathrm{1mm}^{2}$ COMSOL model predicting a total deflection of $304 \mu \mathrm{m}$. (d) A $2 \mathrm{~mm} \times 2 \mathrm{~mm}$ COMSOL model predicting a total deflection of $1261 \mu \mathrm{m}$.

By doubling the length of the actuated segment in the second leg, the actuator continues to grow in total deflection for each new segment added. The value of $L$ that maximized deflection for this configuration was found to be $308 \mu \mathrm{m}$. The analytic model predicts the gain in deflection of the first leg to be $62 \mu \mathrm{m}$ and each leg thereafter to be $27 \mu \mathrm{m}$. Various parameters, such as width of the beam, spacing between serpentines, and the number of serpentines were optimized to obtain a $300 \mu \mathrm{m}$ deflection while minimizing the pliancy of the actuator. Since $\mathrm{V}_{\mathrm{PI}}$ of the actuator is based on the actuated segment, the "pull-in" voltage is similar to the actuators characterized above.

\section{Characterization of "Microstage" Actuators}

Microstage actuators with $2 \mathrm{~mm} \times 2 \mathrm{~mm}$ footprints were fabricated alongside actuators with $1 \mathrm{~mm}^{2}$ footprints. A fully released $1 \mathrm{~mm}^{2}$ Microstage can be seen in Fig. 9(a), and a $2 \mathrm{~mm} x$ $2 \mathrm{~mm}$ Microstage can be seen in Fig. 9(b). The $1 \mathrm{~mm}^{2}$ Microstage measured a total deflection of $290 \mu \mathrm{m}$, in good agreement with the COMSOL prediction of $304 \mu \mathrm{m}$ shown in Fig. 9(c), and the $2 \mathrm{~mm}$ Microstage measured $1243 \mu \mathrm{m}$ in good agreement with the COMSOL prediction of $1261 \mu \mathrm{m}$ shown in Fig. 9(d). Optical deflection measurements for the Microstages were taken at each turn in the serpentine, and the results, shown in Fig. 10 correlate well with the COMSOL model. The average deflection gain per leg was found to be $25.6 \mu \mathrm{m}$, which correlates well with the analytic model prediction of $27 \mu \mathrm{ms}$ per leg.

\section{CONCLUSION}

We have presented a simple novel fabrication process for achieving "zipper" actuation through the use of an SOI and thermal silicon dioxide. The deflection and voltage of the actuators were characterized, and the results correlated well with analytic and FEA (Finite Element Analysis) models. Spiral actuators were investigated and insight into their mechanics was presented. "Micro-stage" zipper actuators were conceptualized, modeled and fabricated to achieve $300 \mu \mathrm{m}$ deflection within a $1 \mathrm{~mm}^{2}$, and 1250 $\mu \mathrm{m}$ deflection within a $2 \mathrm{~mm} \times 2 \mathrm{~mm}$ footprint, in order to enable the fabrication of a microstrip patch antenna.

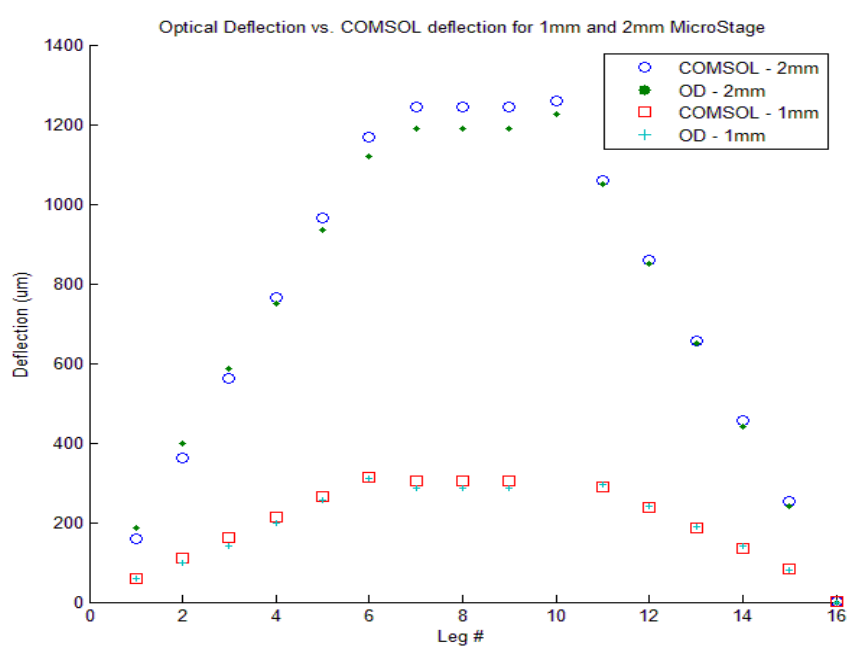

Figure 10: Optical Deflection (OD) measurements at each turn of the Microstage serpentine plotted alongside COMSOL predictions for deflection at each turn. Shown here is the correlation in measurements and predictions for both a $2 \mathrm{~mm}$ and $1 \mathrm{~mm}$ actuator.

\section{ACKNOWLEDGMENT}

Electron microscopy was performed with support from the University of Maryland Nanoscale Imaging, Spectroscopy, and Properties Laboratory (NISPLab).

\section{REFERENCES}

[1] L. Wu and H. Xie, "A Lateral-Shift-Free and Large-VerticalDisplacement Electrothermal Actuator for Scanning Micromirror/Lens," in Transducers \& Eurosensors, Lyons, France, 2007.

[2] T. Fukushige, S. Hata and A. Shimokohbe, "A MEMS Conical Spring Actuator Array," vol. 14, no. 2, 2005.

[3] S. Kwon, V. Milanovic and L. P. Lee, "Large-Displacement Vertical Microlens Scanner," IEEE Photonics Technology Letters, vol. 14, no. 11, 2002.

[4] C. Chang, C.-F. Chiang, C.-H. Liu and C.-H. Liu, "A lobstersniffing-inspired method for micro-objects manipulation using electrostatic micro-actuators," J. Micromech. Microeng., vol. 15,2005 .

[5] N. Dhaubanjar, "The Design and Analysis of Optical Scanners for Optical Coherence Tomography," University of Texas at Arlington, Arlington, Texas, 2006.

[6] A. K. Chinthakindi and P. A. Kohl, "Electrostatic Actuators with Intrinsic Stress Gradient," Journal of The Electrochemical Society, vol. 149, no. 8, 2002.

[7] N. Dhaubanjar, S. M. N. Rao, Y. Cai, D. Popa, M. Chiao and J.-C. Chiao, "A Cantilever-type Electrostatic Zipping Actuator," Smart Structures, Devices, and Systems III, vol. 6414, 2007.

[8] M. A. Rosa, D. De Bruyker, A. R. Volkel, E. Peeters and J. Dunec, "A novel external electrode configuration," Journal of Micromechanics and Microengineering, vol. 14, pp. 446-451, 2004.

[9] R. Legtenberg, J. Gilbert, S. D. Senturia and M. Elwenspoek, "Electrostatic Curved Electrode Actuators," vo. 6, no. 3, 1997. 